\title{
Beyond the adrenals: Organ manifestations in inherited primary adrenal insufficiency in children
}

\section{Clemens Kamrath}

Division of Pediatric Endocrinology and Diabetology, Center of Child and Adolescent Medicine, Justus Liebig University, Giessen, Germany
Correspondence should be addressed to C Kamrath Email

clemens.kamrath@paediat.m ed.uni-giessen.de

\begin{abstract}
Primary adrenal insufficiency (PAI) in children is mostly due to genetic defects. The understanding of the molecular genetics of the causes of adrenal insufficiency in the pediatric population has made significant progress during the last years. It has been shown that inherited PAI can lead to certain clinical manifestations and health problems in children beyond the adrenals. Organ dysfunctions associated with different forms of PAI in children include a wide range of organs such as gonads, brain, heart, bone, growth, bone marrow, kidney, skin, parathyroid, and thyroid. Diagnosing the correct genetic cause of PAl in children is therefore crucial to adequately control long-term treatment and follow-up in such patients.
\end{abstract}

Primary adrenal insufficiency (PAI) in children is a rare and life-threatening disease caused by the inability of the adrenal cortex to produce sufficient amounts of glucocorticoids and/ or mineralocorticoids. Up to $90 \%$ of cases of PAI in adults in Western countries are caused by autoimmune destruction of the adrenal cortex (1). In contrast, PAI is caused mostly due to genetic defects in children $(2,3)$.

The understanding of the molecular genetics of the causes of adrenal insufficiency in the pediatric population has made significant progress $(4,5)$.

Diagnosing the correct genetic cause of PAI in children is crucial because of certain comorbidities and different forms of inheritance in the various subtypes of PAI. Most forms of PAI are inherited autosomal recessive, but some are inherited X-linked or show an imprinted mode of inheritance. Accurate molecular diagnosis is important not only to properly inform the family about the likelihood of a future pregnancy also being affected, but also to discover affected, but clinically asymptomatic mutation carriers, in the family.

Beside the need for hydrocortisone and fludrocortisone replacement, different causes of PAI are associated with a wide range of extra-adrenal organ manifestations. Kallali et al. have reported in this issue of EJE that development of testicular adrenal rest tumors (TART) in a male suffering from PAI of unknown cause had led to suspicion of tumor and unnecessary orchidectomy (6). Therefore, the correct molecular diagnosis is important to prevent patients from unnecessary procedures and to offer the right treatment; in this case fertility protection such as sperm preservation or intensified glucocorticoid treatment. Importantly, inherited PAI can lead to certain clinical manifestations and health problems in children beyond the adrenals. Organ dysfunctions associated with inherited forms of PAI in children include gonads, brain, heart, bone, growth, bone marrow, kidney, skin, parathyroid, and thyroid.

\section{Gonadal dysfunction}

Deficient or reduced fetal testicular steroidogenesis results in phenotypically normal female genitalia or undervirilized male genitalia in patients with a $46, \mathrm{XY}$ karyotype $(46, \mathrm{XY}$ disorder of sex development (DSD)) in the subsequent CAH

Published by Bioscientifica Ltd. 
forms: classic congenital lipoid adrenal hyperplasia due to mutations in the STAR gene, P450 side chain cleavage enzyme deficiency (P450scc deficiency) due to mutations in the CYP11A1 gene, 17 $\alpha$-hydroxylase deficiency, $3 \beta$ hydroxysteroid dehydrogenase (-HSD) deficiency, and P450 oxidoreductase (POR) deficiency. Increased androgen production from the fetal adrenal resulting in 45,XX DSD occurs in classic 21 -hydroxylase deficiency, $11 \beta$-hydroxylase deficiency, 3 $\beta$-HSD deficiency, and POR deficiency (7). Syndromic diseases with PAI and undervirilization in males include the IMAGe syndrome and the MIRAGE syndrome (both discussed in more detail in the subsequent section). Development of TART with reduced fertility in men has been reported in classic 21-hydroxylase deficiency,

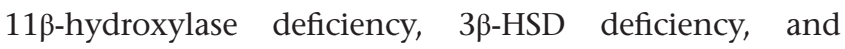
nonclassic forms of lipoid CAH or P450scc deficiency (5). Importantly, development of TART has been described in non-CAH cases, such as in patients with mutations in the nicotinamide nucleotide transhydrogenase (NNT) gene encoding an NADPH-producing enzyme of the inner mitochondrial membrane (8) (discussed in more detail in the section on 'Cardiomyopathies').

$\mathrm{X}$-linked congenital adrenal hypoplasia (X-AHC) is due to mutations in the NROB1 gene, which causes a loss of function in the nuclear receptor protein DAX-1. X-AHC leads to the development of PAI within the first few weeks of life or in childhood. The development of the external male reproductive organs is not disturbed. However, hypogonadotropic hypogonadism arises later in life and the affected men develop azoospermia (9).

Autoimmune primary gonadal insufficiency that leads to hypergonadotropic hypogonadism can occur in patients with autoimmune polyglandular syndrome (APS) type 1 due to mutations in the AIRE gene (discussed in more detail in the subsequent section).

\section{Brain involvement}

The most impressive example is the cerebral involvement of boys suffering from X-linked adrenoleukodystrophy (X-ALD). X-ALD is caused by mutations in the ABCD1 gene that leads to the accumulation of very long chain fatty acids (VLCFA). Adrenal insufficiency affects approximately $70 \%$ of male patients with X-ALD and is often the first clinical symptom. About $50 \%$ of patients develop inflammatory demyelination of the brain. Boys suffering from X-ALD who have PAI without neurological involvement must be closely screened for the onset of cerebral inflammation. Therapeutic options include prophylactic therapy of the cerebral inflammatory demyelinating course in asymptomatic boys with Lorenzo's oil and hematopoietic stem cell transplantation at a very early stage of the cerebral involvement to prevent rapid progression $(10,11)$.

The Triple A (Allgrove) syndrome is an autosomal recessive disorder caused by mutations in the $A A A S$ gene that encodes the ALADIN protein. Triple A syndrome is characterized by the combination of alacrimia, achalasia, PAI, autonomic dysfunction, and neurodegeneration. Alacrimia is often the earliest and most common symptom and often begins in the first months of life. The achalasia of the cardia occurs in about $75 \%$ of patients and is usually the reason for further diagnostic clarification. PAI usually develops after the first two symptoms in the first decade of life, but in some cases may also lead to the diagnosis of the disease through hypoglycemia and seizures. In addition to glucocorticoid deficiency, mineralocorticoid insufficiency is present in up to $15 \%$ of cases (12).

Recently, loss-of-function mutations in the sphingosine-1-phosphate lyase gene (SGPL1) have been discovered in patients suffering from early onset PAI and steroid-resistant nephrotic syndrome. Some cases also developed neurological symptoms such as neurodegenerative diseases, including progressive motor and cognitive decline with ataxia and sensorineural hearing loss (13). The renal phenotype will be presented in more detail in the section on 'Nephrotic syndrome'.

\section{Cardiomyopathies}

Cardiomyopathies have been described in patients with mutations in the NNT gene and in those with mutations in the mitochondrial thioredoxin reductase gene TXNRD2. These genetic defects underscore the important role of oxidative stress and reactive oxygen species detoxification systems in maintaining normal adrenal function. Homozygous or compound-heterozygous mutations in the NNT gene - which encodes an NADPH-producing enzyme of the inner mitochondrial membrane - have been detected in patients with familiar glucocorticoid deficiency (FGD) (14), and nowadays more and more patients with this defect are recognized and described $(8,15)$. Importantly, in contrast to the classic forms of FGD, a substantial number of patients affected by NNT gene mutations also have mineralocorticoid deficiency. Beside adrenal insufficiency, additional organ dysfunction may occur, such as hypothyroidism, cryptorchidism, precocious puberty, TART, azoospermia, and hypertrophic cardiomyopathy. A careful follow-up is 
needed to evaluate mineralocorticoid biosynthesis extent and gonadal, heart, and thyroid function (15).

Thioredoxin reductases are essential proteins for regulating cellular redox balance and reducing damage caused by reactive oxygen species. Recently, homozygous mutations of the TXNRD2 gene could be detected by exome sequencing in consanguineous patients with isolated glucocorticoid deficiency (16). Moreover, inactivating mutations in the TXNRD2 gene have been described in patients suffering from dilated cardiomyopathy (17).

\section{Nephrotic syndrome}

Mutations in the SGPL1 gene are very rare and usually lead to PAI in infancy. Most cases had isolated glucocorticoid deficiency necessitating hydrocortisone replacement, but in some cases mineralocorticoid deficiency was present. The nephrotic syndrome manifested between the ages of 1.5 months and 5.5 years in more than half of the cases. It was steroid resistant and due to focal segmental glomerulosclerosis. Other features of this disease included ichthyosis, which was present in most cases, primary hypothyroidism, neurological symptoms, and cryptorchidism (13).

\section{Bone and growth disorders}

Mutations in the ACTH receptor gene $M C 2 R$ account for $25 \%$ of cases of FGD (FGD type 1). Patients with FGD type 1 are usually quite large and manifest in early infancy and toddlerhood (median at about 2 years). In contrast, FGD type 2 patients, who have mutations in the melanocortin2-receptor-accesory protein gene (MRAP), accounting for $15-20 \%$ of FGD cases, are of average length and manifest earlier during neonatal or infancy period (18).

Growth restriction and bone dysplasias are seen in patients with syndromic forms of PAI. These include the IMAGe syndrome and the MIRAGE syndrome (discussed in more detail in the subsequent section). The IMAGe syndrome is characterized by intrauterine growth retardation (IUGR), metaphyseal dysplasia, adrenal insufficiency, and genital anomalies. Skeletal abnormalities mostly involve delayed bone age and short stature, but may occasionally include metaphyseal and epiphyseal dysplasia. Adrenal insufficiency occurs typically in the first month of life. Genital abnormalities in males include cryptorchidism, micropenis, and hypospadias. IMAGe syndrome is due to autosomal-dominant gain-of-function mutations of the maternally inherited allele of the cyclindependent kinase (CDK)-inhibitor 1C (CDKN1C) gene (19).

\section{Bone marrow dysfunction and immune deficiency}

The MIRAGE syndrome is a recently described multisystem disorder consisting of a core set of phenotypes: myelodysplasia, life-threatening and recurrent infections, restriction of growth, adrenal hypoplasia with insufficiency, genital abnormalities with undervirilisation (46,XY DSD), and enteropathy. Additional associated features include prematurity, chronic lung disease, developmental delay, dysmorphic features, and CNS abnormalities. The syndrome is caused by de novo gainof-function mutations in the gene encoding sterile alpha motif domain-containing protein 9 (SAMD9) (20).

\section{Combined disorders of the endocrine glands}

Autoimmune polyglandular syndrome (APS) type 1 is also referred to as autoimmune polyendocrinopathy candidiasis ectodermal dystrophy (APECED) and is caused by inactivating mutations of the AIRE gene. In addition to the autoimmune PAI, the disease is characterized by chronic mucocutaneous candidiasis in combination with hypoparathyroidism. Chronic candidiasis is usually the first symptom in early childhood and hypoparathyroidism usually occurs in later childhood, as well as adrenal insufficiency. However, about 5\% of patients show autoimmune adrenal insufficiency as the first symptom. Importantly, APS type 1 can be associated with additional autoimmune phenomena such as alopecia, vitiligo, gastritis with or without pernicious anemia, hemolytic anemia, and chronic diarrhea or hypergonadotropic hypogonadism. The severity and the temporal appearance of the symptoms is highly variable, which makes the diagnosis difficult.

\section{Skin manifestation}

In addition to the increased pigmentation of the skin in PAI, a skin manifestation can occur in APS type 1 by chronic mucocutaneous candidiasis, alopecia, and vitiligo, as well as ichthyosis in patients with SGPL1 gene mutations. 
Taken together, achieving a molecular diagnosis has important implications for family counselling, presymptomatic diagnosis, personalized treatment (e.g. mineralocorticoid replacement), and the prediction and treatment of comorbidities such as neurological disorders, growth disorders, fertility disorders (e.g. TART, gonadal insufficiency), and heart diseases. Proper diagnosis of unknown cases of PAI is therefore important to adequately control long-term treatment and follow-up in such patients $(2,3,4,5)$.

\section{Declaration of interest}

The author declares that there is no conflict of interest that could be perceived as prejudicing the impartiality of this commentary.

\section{Funding}

This research did not receive any specific grant from any funding agency in the public, commercial, or not-for-profit sector.

\section{References}

1 Charmandari E, Nicolaides NC \& Chrousos GP. Adrenal insufficiency. Lancet 2014383 2152-2167. (https://doi.org/10.1016/S01406736(13)61684-0)

2 Tsai SL, Green J, Metherell LA, Curtis F, Fernandez B, Healey A \& Curtis J. Primary adrenocortical insufficiency case series: genetic etiologies more common than expected. Hormone Research in Paediatrics 201685 35-42. (https://doi.org/10.1159/000441843)

3 Wijaya M, Huamei M, Jun Z, Du M, Li Y, Chen Q, Chen H \& Song G. Etiology of primary adrenal insufficiency in children: a 29-year singlecenter experience. Journal of Pediatric Endocrinology and Metabolism 201932 615-622. (https://doi.org/10.1515/jpem-2018-0445)

4 Amano N, Narumi S, Hayashi M, Takagi M, Imai K, Nakamura T, Hachiya R, Sasaki G, Homma K, Ishii T et al. Genetic defects in pediatric-onset adrenal insufficiency in Japan. European Journal of Endocrinology 2017177 187-194. (https://doi.org/10.1530/EJE-17-0027)

5 Narumi S. Rare monogenic causes of primary adrenal insufficiency. Current Opinion in Endocrinology, Diabetes, and Obesity 201825 172-177. (https://doi.org/10.1097/MED.0000000000000401)

6 Kallali W, Gray E, Mehdi MZ, Lindsay RS, Metherell LA, Buonocore F, Suntharalingham JP, Achermann J \& Donaldson M. Long-term outcome of partial P450 side-chain cleavage enzyme deficiency in three brothers: the importance of early diagnosis. European Journal of Endocrinology 2020 182 K15-K24. (https://doi.org/10.1530/EJE-19-0696)

7 Engels M, Span PN, van Herwaarden AE, Sweep FCGJ, Stikkelbroeck NMML \& Claahsen-van der Grinten HL. Testicular adrenal rest tumors: current insights on prevalence,characteristics, origin and treatment. Endocrine Reviews 201940 973-987. (https:// doi.org/10.1210/er.2018-00258)

8 Hershkovitz E, Arafat M, Loewenthal N, Haim A \& Parvari R. Combined adrenal failure and testicular adrenal rest tumor in a patient with nicotinamide nucleotide transhydrogenase deficiency. Journal of Pediatric Endocrinology and Metabolism 201528 1187-1190. (https://doi.org/10.1515/jpem-2015-0075)
9 Frapsauce C, Ravel C, Legendre M, Sibony M, Mandelbaum J, Donadille B, Achermann JC, Siffroi JP \& Christin-Maitre S. Birth after TESE-ICSI in a man with hypogonadotropic hypogonadism and congenital adrenal hypoplasia linked to a DAX-1 (NR0B1) mutation. Human Reproduction 201126 724-728. (https://doi.org/10.1093/ humrep/deq372)

10 Moser HW, Raymond GV, Lu SE, Muenz LR, Moser AB, Xu J, Jones RO, Loes DJ, Melhem ER, Dubey P et al. Follow-up of 89 asymptomatic patients with adrenoleukodystrophy treated with Lorenzo's oil. Archives of Neurology 200562 1073-1080. (https://doi. org/10.1001/archneur.62.7.1073)

11 Peters C, Charnas LR, Tan Y, Ziegler RS, Shapiro EG, DeFor T, Grewal SS, Orchard PJ, Abel SL, Goldman AI et al. Cerebral X-linked adrenoleukodystrophy: the international hematopoietic cell transplantation experience from 1982 to 1999. Blood $2004 \mathbf{1 0 4}$ 881-888. (https://doi.org/10.1182/blood-2003-10-3402)

12 Milenkovic T, Zdravkovic D, Savic N, Todorovic S, Mitrovic K, Koehler K \& Huebner A. Triple A syndrome: 32 years experience of a single centre (1977-2008). European Journal of Pediatrics 2010169 1323-1328. (https://doi.org/10.1007/s00431-010-1222-7)

13 Prasad R, Hadjidemetriou I, Maharaj A, Meimaridou E, Buonocore F, Saleem M, Hurcombe J, Bierzynska A, Barbagelata E, Bergadá I et al. Sphingosine-1-phosphate lyase mutations cause primary adrenal insufficiency and steroid-resistant nephrotic syndrome. Journal of Clinical Investigation 2017127 942-953. (https://doi.org/10.1172/ JCI90171)

14 Meimaridou E, Kowalczyk J, Guasti L, Hughes CR, Wagner F, Frommolt P, Nürnberg P, Mann NP, Banerjee R, Saka HN et al. Mutations in NNT encoding nicotinamide nucleotide transhydrogenase cause familial glucocorticoid deficiency. Nature Genetics 201244 740-742. (https://doi.org/10.1038/ng.2299)

15 Roucher-Boulez F, Mallet-Motak D, Samara-Boustani D, Jilani H, Ladjouze A, Souchon PF, Simon D, Nivot S, Heinrichs C, Ronze M et al. NNT mutations: a cause of primary adrenal insufficiency, oxidative stress and extra-adrenal defects. European Journal of Endocrinology 2016175 73-84. (https://doi.org/10.1530/EJE-160056)

16 Prasad R, Chan LF, Hughes CR, Kaski JP, Kowalczyk JC, Savage MO, Peters CJ, Nathwani N, Clark AJ, Storr HL et al. Thioredoxin reductase 2 (TXNRD2) mutation associated with familial glucocorticoid deficiency (FGD). Journal of Clinical Endocrinology and Metabolism 201499 E1556-E1563. (https://doi.org/10.1210/jc.2013-3844)

17 Sibbing D, Pfeufer A, Perisic T, Mannes AM, Fritz-Wolf K, Unwin S, Sinner MF, Gieger C, Gloeckner CJ, Wichmann HE et al. Mutations in the mitochondrial thioredoxin reductase gene TXNRD2 cause dilated cardiomyopathy. European Heart Journal 201132 1121-1133. (https:// doi.org/10.1093/eurheartj/ehq507)

18 Chung TT, Chan LF, Metherell LA \& Clark AJ. Phenotypic characteristics of familial glucocorticoid deficiency (FGD) type 1 and 2. Clinical Endocrinology 201072 589-594. (https://doi.org/10.1111/ j.1365-2265.2009.03663.x)

19 Arboleda VA, Lee H, Parnaik R, Fleming A, Banerjee A, Ferraz-deSouza B, Délot EC, Rodriguez-Fernandez IA, Braslavsky D, Bergadá I et al. Mutations in the PCNA-binding domain of CDKN1C cause IMAGe syndrome. Nature Genetics 201244 788-792. (https://doi. org/10.1038/ng.2275)

20 Narumi S, Amano N, Ishii T, Katsumata N, Muroya K, Adachi M, Toyoshima K, Tanaka Y, Fukuzawa R, Miyako K et al. SAMD9 mutations cause a novel multisystem disorder, Mirage syndrome, and are associated with loss of chromosome 7. Nature Genetics 201648 792-797. (https://doi.org/10.1038/ng.3569)
Received 8 December 2019

Revised version received 21 January 2020

Accepted 23 January 2020 